Vera Lucia Fonseca de Camargo-Neves ${ }^{1}$

Gizelda Katz 2

Lilian Aparecida Colebrusco Rodas 1

Daniela Witacker Poletto 1

Lisete Cruz Lage 2

Roberta Maria Fernandes Spínola 2

Oswaldo Gonçalves Cruz ${ }^{3}$

\section{Utilização de ferramentas de análise espacial na vigilância epidemiológica de leishmaniose visceral americana - Araçatuba, São Paulo, Brasil, 1998-1999}

\author{
Use of spatial analysis tools in the epidemiological \\ surveillance of American visceral leishmaniasis, \\ Araçatuba, São Paulo, Brazil, 1998-1999
}

1 Superintendência de Controle de Endemias, Secretaria de Estado de Saúde de São Paulo. Rua Paula Souza 166, lo andar, São Paulo, SP 01027-000, Brasil. 2 Centro de Vigilância Epidemiológica Professor Alexandre Vranjac, Secretaria de Estado de Saúde de São Paulo. Av. Dr. Arnaldo 351, 60 andar, sala 611, São Paulo, SP 01246-902, Brasil. 3 Programa de Computação Científica, Fundação Oswaldo Cruz. Av. Brasil 4365, Rio de Janeiro, $R J$ 21041-210, Brasil.

\begin{abstract}
The control of American visceral leishmaniasis (AVL) is based on combating the vector and eliminating the domestic reservoir of the focus area-defined as 200 meters around human or canine cases. This paper discusses the use of spatial analysis techniques in the epidemiological surveillance of AVL in Araçatuba, São Paulo State, in order to propose a model for territorial epidemiological surveillance, reformulating current control strategies. The results showed that AVL transmission was not homogeneous; human cases were more frequent in areas with higher canine prevalence rates. Vector dispersion appeared to be restricted to a few houses, although it was not possible to model the vector density. In order to study the vector distribution and correlated covariates, a field study based on house sampling is being conducted. The results will aid the development of new spatial analysis tools and possibly redefine protocols and routines for the control of this endemic disease in urban areas.
\end{abstract}

Key words Spatial Analysis; Geographic Information System; Visceral Leishmaniasis; Lutzomyia longipalpis

Resumo O controle da Leishmaniose Visceral Americana (LVA) está fundamentado no combate ao vetor e na eliminação do reservatório doméstico da área de foco - 200 metros em torno do caso humano ou canino. O presente trabalho tem como objetivo discutir a utilização de técnicas de análise espacial na vigilância epidemiológica da LVA no Município de Araçatuba, São Paulo, buscando estabelecer um modelo de vigilância epidemiológica em base territorial, redirecionando as estratégias de controle atualmente adotadas. Verificou-se que a transmissão da LVA não foi homogênea no município: a transmissão humana ocorreu nas áreas com maiores taxas de prevalência canina. A dispersão do vetor parece restrita a poucos domicílios, embora não tenha sido possível estabelecer um perfil da densidade vetorial. Visando estudar a distribuição do vetor e variáveis correlacionadas está sendo realizado estudo de campo por amostragem de domicílios, que permitirá o desenvolvimento de novas ferramentas de análise espacial e, possivelmente, permitirá redefinir as propostas de controle da endemia em ambiente urbano.

Palavras-chave Análise Espacial; Sistema de Informação Geográfica; Leishmaniose Visceral Americana; Lutzomyia longipalpis 


\section{Introdução}

No Estado de São Paulo, casos importados de leishmaniose visceral americana (LVA), também chamada de Calazar, são detectados todos os anos. Data da década de 1970 o primeiro registro da ocorrência do agente transmissor - Lutzomyia longipalpis - em região restrita, formada pela extensão da Serra da Mantiqueira, a leste do Estado, na qual, até o presente, não existem registros de transmissão autóctone da doença (Forattini et al., 1970). A partir de 1997, este vetor foi identificado na Região Oeste do Estado, onde sua presença era desconhecida, seguida do encontro da infecção por Leishmania chagasi em cães e, posteriormente, em 1999, a ocorrência de casos humanos. Desde então, medidas de controle vêm sendo implementadas, visando reduzir a morbimortalidade humana por LVA no Estado (SES-SP, 2000).

O presente trabalho tem como objetivo discutir a utilização de técnicas de análise espacial na vigilância epidemiológica da LVA no Município de Araçatuba, buscando estabelecer mecanismos de vigilância de base territorial, de forma a propor recomendações que possam direcionar as medidas de vigilância e controle adotadas.

\section{Metodologia}

O Município de Araçatuba está localizado na Região Noroeste do Estado de São Paulo, em área total de $1.330 \mathrm{~km}^{2}$, cuja área urbana abrange $59,7 \mathrm{~km}^{2}$, na qual se distribuem 162.546 habitantes e em que há 62.000 imóveis. O abastecimento de água abrange $98 \%$ das residências, enquanto $97 \%$ dispõem da cobertura de rede de esgotos e $80 \%$, da coleta de lixo. A atividade econômica do município está relacionada à agropecuária e à indústria. A população canina estimada foi de 33.015 cães. A área urbana está dividida em oito áreas, das quais sete são subdivididas em cinco setores, conforme critério do Programa de Erradicação do Aedes aegypti (PEAe). Portanto, para este trabalho, foram considerados os 36 setores do município.

A vigilância epidemiológica de casos humanos vem sendo realizada através da notificação de casos suspeitos, de sua investigação e da confirmação mediante exame parasitológico direto ou por critério clínico epidemiológico. $\mathrm{O}$ censo sorológico canino foi efetuado no ano de 1999, quando foram examinados 30.291 cães. As taxas de prevalência sorológica canina foram calculadas por setor.
A pesquisa entomológica foi feita pelo Serviço Regional de Araçatuba da Superintendência de Controle de Endemias (SUCEN) no período de agosto a novembro de 1998 e de junho a agosto de 1999. Em um primeiro momento, as coletas foram realizadas de julho a novembro de 1998 com base em notificação de insetos incômodos, de caso humano ou de cão suspeito de LVA. Posteriormente, de fevereiro a setembro de 1999, a coleta esteve dirigida a alguns setores em que havia caso humano e/ou canino confirmado. Foram preconizadas pesquisas entomológicas nos domicílios de residência dos cães e/ou de casos humanos suspeitos ou confirmados, bem como naqueles com notificação de insetos incômodos, estendendo-se a investigação a mais cinco residências do quarteirão ou próximo a ele. Em cada residência, foram pesquisados: as paredes do intra e peridomicílio, os abrigos de animais e os possíveis locais de repouso do flebotomíneo. O período da captura foi de uma hora após o crepúsculo vespertino até três horas. Para a coleta dos exemplares foram utilizados aspiradores manuais elétricos.

Para a análise espacial considerou-se cada setor do PEAe como unidade de agregação, empregando-se o MapInfo 5.0 (MapInfo Corporation, 1998). A base cartográfica, que fazia parte do material de preparação do Censo 2000, foi cedida, em formato CAD, pela Fundação Instituto Brasileiro de Geografia e Estatística (IBGE). Foram construídas várias camadas de informação que envolviam casos humanos, de cães e a presença de flebotomíneos, cada uma podendo ser representada mediante diversos indicadores. Neste trabalho utilizou-se o número absoluto de casos humanos em cada setor, a proporção de cães positivos, o número absoluto de domicílios com presença do vetor e o número de vetores capturados em cada setor.

\section{Resultados}

No período de 1998 a 1999 foram confirmados 15 casos humanos em Araçatuba, empregando-se, como critérios de confirmação, o encontro do parasita e o clínico-epidemiológico. A faixa etária mais acometida foi a de 0 a 4 anos $(40,0 \%)$, tendo ocorrido $53,3 \%$ dos casos em indivíduos menores de 15 anos.

Em Araçatuba, o inquérito canino censitário foi realizado conforme preconizado pela Fundação Nacional de Saúde (MS, 1999), mostrando prevalência geral de $12,1 \%$ e variando entre $4,1 \%$ a $25,8 \%$ nos setores do município. Em 54,0\% dos exames coletados em Araçatuba, 
o tempo entre a coleta e o retorno do resultado variou de 60 a 90 dias.

A Figura 1 mostra a distribuição do número absoluto de casos humanos sobreposta ao indicador de infecção canina. Observa-se maior concentração de casos nos setores a leste e ao norte do município, setores nos quais foram detectados casos humanos e as maiores taxas de prevalência de cães sorologicamente positivos.

Os resultados da captura do vetor estão na Figura 2, na qual se procurou verificar a positividade das residências e, também, a densidade de L. longipalpis, considerando que o primeiro indicador permite dimensionar a extensão territorial do problema e o segundo, a intensidade da infestação.

As capturas entomológicas foram efetuadas em 798 domicílios, situados em 24 dos 36 setores existentes no Município de Araçatuba. Cabe ressaltar que, em alguns setores, a borrifação com inseticida de ação residual já havia sido implementada em razão da ocorrência de caso humano de LVA. O número de imóveis pesquisados por setor variou de 1 a 105 domicílios. Em cinco setores, as capturas foram negativas. O número de exemplares capturados variou de 1 a 179. Não se verificou distribuição homogênea do número de flebotomíneos pelo município.

A distribuição espacial que relaciona o número de imóveis positivos e o número de exemplares capturados pode ser observada na Figura 2. Não se evidenciou associação entre o número de imóveis positivos e o maior número de L. longipalpis capturados. As maiores densidades, assim como as maiores médias de exemplares por domicílio pesquisado, foram observadas nos setores centro e leste do município.

\section{Discussão}

A análise de dados distribuídos pelo espaço geográfico vem sendo cada vez mais valorizada na gestão de saúde, por apontar novos subsídios para o planejamento e a avaliação das ações baseadas na análise da distribuição espacial das doenças, localização dos serviços de saúde e dos riscos ambientais, entre outros (Barcellos \& Bastos, 1996, 1997). No caso em estudo, a integração de diferentes camadas de informação abriu vastas possibilidades, além daquelas apresentadas, nas quais ambiente, vetor e reservatório compõem o cenário de risco para a ocorrência de infecção humana.

Algumas técnicas de análise estão sendo implementadas - em particular, a construção de superfícies por interpolação - de forma a es-

\section{Figura 1}

Distribuição do número absoluto de casos humanos e da proporção de cães soropositivos. Araçatuba, São Paulo, 1998-1999.
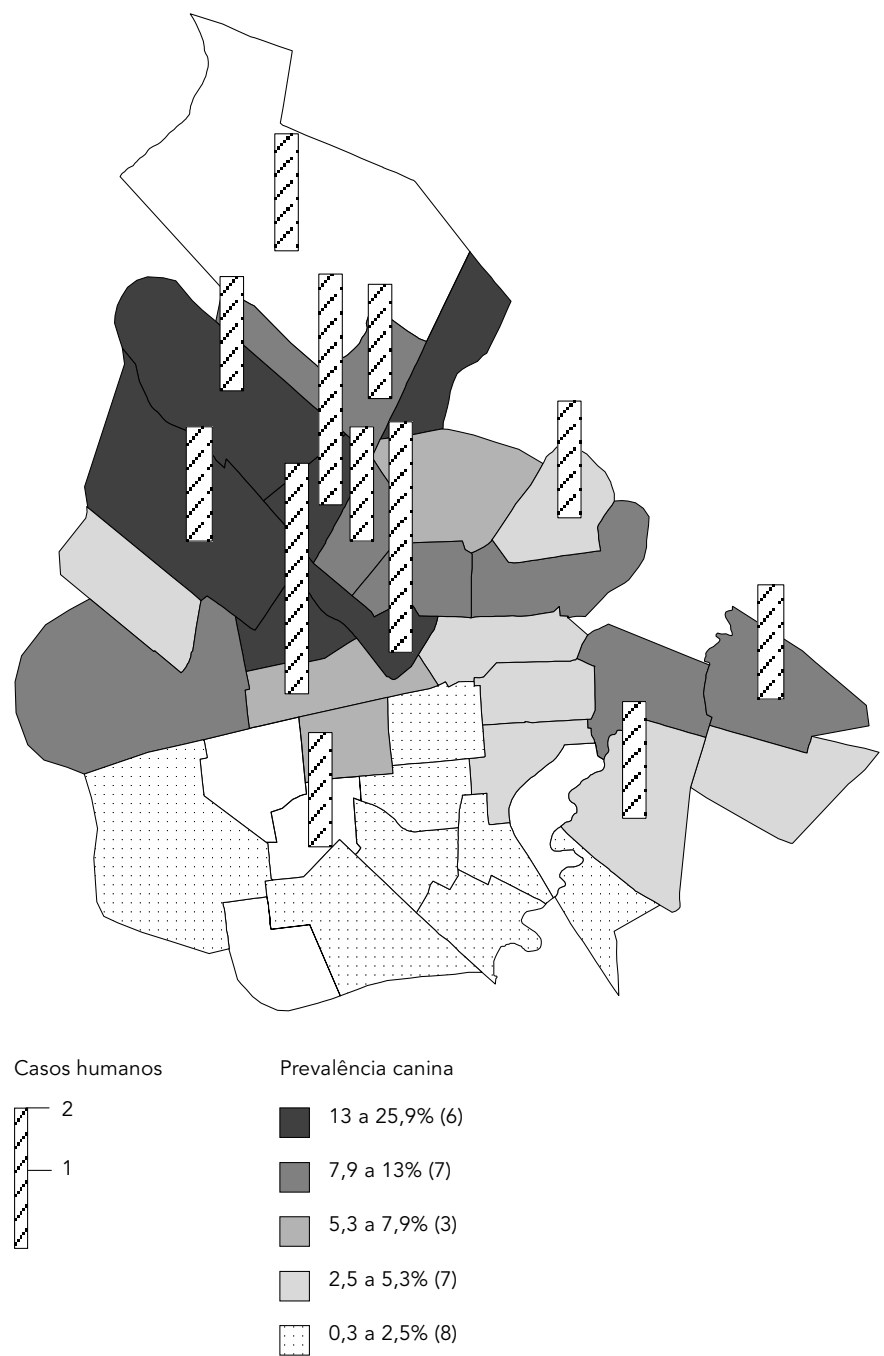

tabelecer um gradiente contínuo na distribução de cada indicador, fora dos limites estabelecidos pela divisão setorial. É evidente que vetor e reservatório não respeitam as linhas arbitrárias da divisão de áreas administrativas. Entretanto, ainda que versões recentes de Sistema de Informações geográficas (SIGs) permitam a criação de grade interpolada, o modelo disponível - em geral, apenas um alisamente não paramétrico - não é necessariamente o mais adequado.

Neste trabalho buscou-se detectar um padrão de distribuição da LVA de modo a propor 
Figura 2

Distribuição da proporção de domicílios positivos para a presença de Lutzomyia longipalpis e do número de exemplares capturados. Araçatuba, São Paulo, 1998-1999.

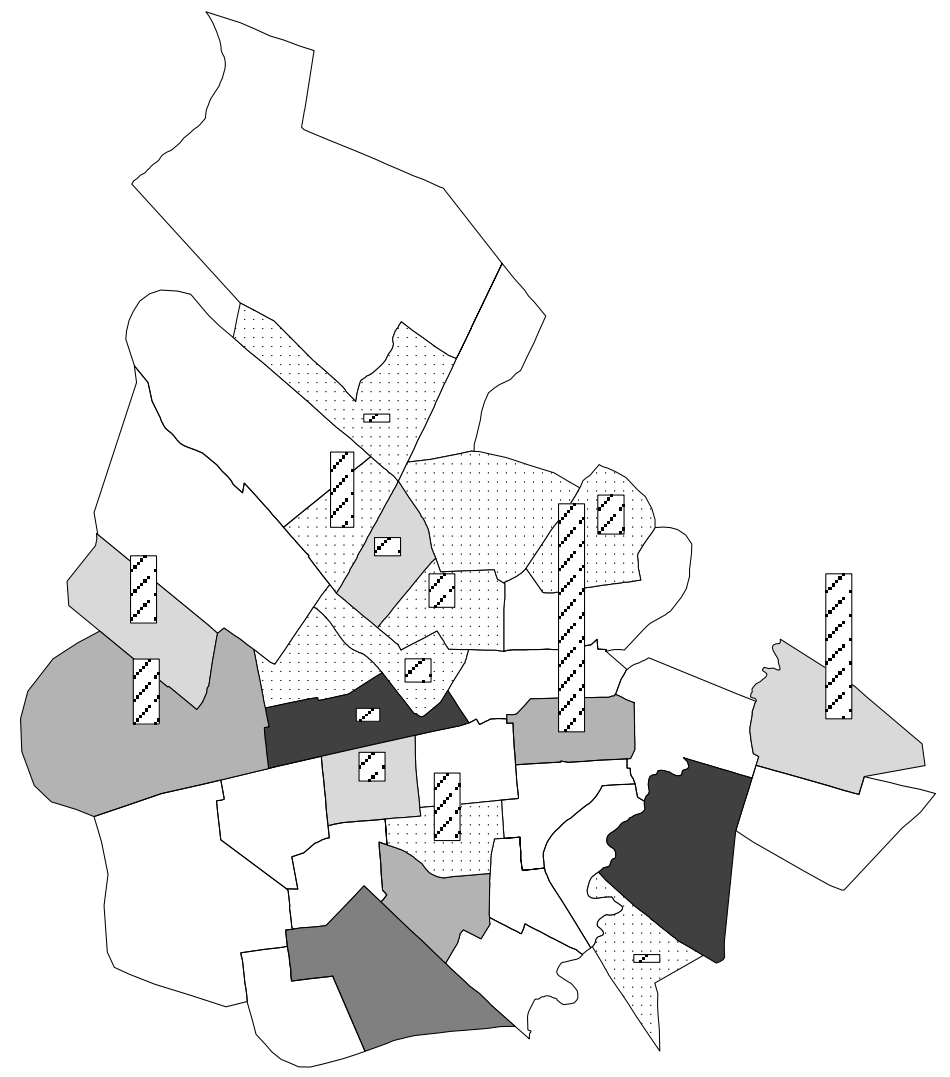

Número de exemplares capturados

$b^{140}-70$

Proporção de domicílios positivos

70 a $100 \%(2)$

40 a $70 \%(1)$

20 a $40 \%$ (3)

10 a $20 \%(4)$

0 a $10 \%(9)$

medidas preventivas. A aspersão com inseticidas de ação residual associada à eliminação de cães infectados são as principais medidas preconizadas pelo Ministério da Saúde (MS, 1999) para o controle da doença. Entende-se que apenas a execução destas ações isoladas sem análise sistemática que possibilite avaliar o impacto de tais medidas, acaba por torná-las inócuas.

A Superintendência de Controle de Epidemias (SUCEN) - órgão responsável pelo controle vetorial no Estado de São Paulo - vem ado- tando medidas de controle químico contra o vetor apenas na ocorrência de casos humanos confirmados, em área denominada "foco", que cobre 200 metros em torno. A análise espacial dos dados apresentados neste trabalho indica a necessidade de estudos mais aprofundados, com o recurso a métodos que permitam redimensionar o "foco" e, possivelmente, repensar o critério, centrado apenas nos casos humanos confirmados.

O geo-referenciamento dos dados permitiu mostrar correlação entre os setores em que há ocorrência de infecção humana e aqueles com altas taxas de prevalência canina, tal como destes com os setores em que existem densidades mais elevadas de L. longipalpis, ainda que em base apenas visual, sem que fosse testada esta associação. Mesmo assim, pode-se estabelecer, por intermédio dos mapas, regiões potenciais de maior risco de ocorrência da doença, para as quais deverão ser orientadas as medidas de controle preventivas, como, por exemplo, o exame sorológico dos cães e a eliminação daqueles sorologicamente positivos, somadas às medidas voltadas para o vetor, como a borrifação dos domicílios com inseticidas de ação residual nos meses propícios ao aumento de sua densidade.

A grande variabilidade da distribuição dos flebotomíneos nos setores sugere que sua presença está restrita a poucos domicílios, apontando para um padrão de distribuição focal. $\mathrm{O}$ padrão irregular indica a necessidade de estudar o comportamento do vetor com resolução maior do que setor. Em função disso, está em desenvolvimento um estudo de distribuição de flebotomíneos em cinco setores do Município de Araçatuba, no qual o desenho da amostra, tendo a quadra como unidade de resolução espacial, considerou a necessidade de analisar a superfície de densidade do vetor e de correlacioná-la com aspectos ambientais dos peridomicílios, tais como a presença de vegetação, raízes e troncos de árvores e a matéria orgânica no solo - representando os possíveis abrigos e criadouros do vetor - e a presença, além do cão, de outros animais domésticos, como galinhas, coelhos e outros - representando outras fontes de alimento para o vetor.

Um aspecto a ser ressaltado é que a construção de um SIG voltado à vigilância epidemiológica, que envolva diversos dados e em diferentes escalas, não é tarefa fácil, e os problemas metodológicos estão longe de uma solução padrão. O dado geo-referenciado para este tipo de problema não está facilmente disponível; as informações cartográficas ainda são de difícil obtenção apesar dos recentes esforços 
do Ministério da Saúde e da Organização PanAmericana de Saúde (OPAS) (Carvalho et al., 2000); por fim, os métodos de análise, mesmo os modelos mais simples de interpolação, ainda não estão ao alcance do usuário das Secretarias de Saúde. A experiência que está sendo acumulada no estudo e controle da epidemia de Leishmaniose Visceral em Araçatuba pode contribuir substancialmente para o desenvolvimento das ferramentas de análise espacial voltada para vigilância epidemiológica.

\section{Referências bibliográficas}

BARCELLOS, C. \& BASTOS, F. I., 1996. Geoprocessamento, ambiente e saúde: Uma união possível? Cadernos de Saúde Pública, 12:389-397.

BARCELLOS, C. \& SANTOS, M. S., 1997. Colocando dados no mapa: A escolha da unidade espacial de agregação e integração de bases de dados em saúde e ambiente através do geoprocessamento. Informe Epidemiológico do SUS, 6:21-29.

CARVALHO, M. S.; PINA, M. F. \& SANTOS, S. M., 2000. Conceitos Básicos de Sistemas de Informações Geográficas e Cartografia Aplicados à Saúde. Brasília: Organização Panamericana de Saúde/Ministério da Saúde.
FORATTINI, O. P.; RABELO, E. X. \& PATTOLI, D. G. B., 1970. Sobre o encontro de Lutzomyia longipalpis (Lutz \& Neiva) no Estado de São Paulo. Revista de Saúde Pública, 4:99-100.

MAPINFO CORPORATION, 1998. Mapinfo Professional, Version 5.0. New York: Troy.

MS (Ministério da Saúde), 1999. Controle, Diagnóstico e Tratamento da Leishmaniose Visceral (Calazar) - Normas Técnicas. Brasília: Fundação Nacional de Saúde, Ministério da Saúde.

SES-SP (Secretaria de Estado da Saúde de São Paulo), 2000. Leishmaniose Visceral Americana - Informe Técnico. São Paulo: SES-SP. 